\title{
Спектр энергий и радиационные характеристики экситонов в квантовых ямах различной ширины
}

\section{Белов П.А.}

Санкт-Петербургский государственный университет, 199034, Санкт-Петербург, Университетская наб., 7/9

DOI 10.34077/Semicond2019-155

Состояния экситона и свето-экситонное взаимодействие в гетероструктурах с квантовыми ямами (КЯ) исследовались экспериментально на протяжении нескольких десятилетий [1]. Тем не менее, только к настоящему времени параметры выращиваемых структур стали сравнимыми с точностью теоретического моделирования [2]. Более того, недавние измерения спектров отражения высококачественных гетероструктур показывают, что нерадиационное уширение ансамбля экситонов может быть порядка или даже меньше радиационного [3]. Таким образом, высокое качество выращиваемых гетероструктур требует повышения точности моделирования состояний и времен жизни экситона.

В настоящем докладе, мы представляем результаты модельно-точного микроскопического моделирования основного и возбужденных состояний экситона в одиночной полупроводниковой КЯ, а также скоростей радиационного распада этих состояний [4,5]. Мы рассматриваем трехмерное уравнение Шредингера для экситона в КЯ и аналитически определяем спектр соответствующего дифференциального оператора. В частности, определяем энергии связанных состояний электрондырочных пар и квазисвязанных состояний (резонансов). Мы классифицируем связанные состояния и резонансы по типам их доминирующих одномерных квантоворазмерных состояний и относительного движения электрона и дырки в плоскости КЯ [6].

Основываясь на теоретическом анализе спектра, мы вычисляем и идентифицируем состояния тяжелого и легкого экситона для различных ширин КЯ на основе $\mathrm{Al}_{\mathrm{x}} \mathrm{Ga}_{1-\mathrm{x}} \mathrm{As} / \mathrm{GaAs}$ и GaAs/ $\mathrm{In}_{\mathrm{x}} \mathrm{Ga}_{1-\mathrm{x}} \mathrm{As}$. Вычисленные уровни энергии непрерывно зависят от ширины КЯ как от внешнего параметра. Это позволяет нам пронаблюдать изменение положения уровней энергии при переходе от модели двумерного экситона в узкой КЯ к модели квантования экситона как целого в широкой КЯ. При этом наблюдаются пересечения (квазипересечения) уровней разной (одинаковой) симметрии квантоворазмерных состояний. Объяснение этих эффектов дается на основе симметрийных свойств волновой функции. Наш метод вычисления волновой функции асимптотически точен. Он учитывает различие эффективных масс и диэлектрических проницаемостей в КЯ и в барьере [7]. Более того, наш метод позволяет с высокой точностью моделировать состояния экситона в асимметричных КЯ [8].

Мы вычисляем скорости радиационного распада найденных состояний, используя модель свето-экситонного взаимодействия Е.Л. Ивченко [1]. Теоретические результаты сопоставляются с оригинальными экспериментальными спектрами отражения гетероструктур с КЯ на основе InGaAs/GaAs. Радиационные и нерадиационные уширения как основного, так и возбужденных состояний определяются из фитирования спектров и сравниваются с вычисленными значениями. Наблюдается хорошее согласие теоретических и экспериментальных результатов.

Финансовая поддержка предоставлена Российским Научным Фондом, грант 19-72-20039.

[1] E.L. Ivchenko, Optical spectroscopy of semiconductor nanostructures, Alpha Sci., Harrow (2005).

[2] S.V. Poltavtsev, Y.P. Efimov, Y.K. Dolgikh et al., Solid State Comm., 199, 47 (2014).

[3] A.V. Trifonov, S.N. Korotan, A.S. Kurdyubov et al., Phys. Rev. B., 91, 115307 (2015).

[4] E.S. Khramtsov, P.A. Belov, P.S. Grigoryev et al., J. Appl. Phys., 119, 184301 (2016).

[5] P.A. Belov, Semiconductors, 52, 551 (2018).

[6] P.A. Belov, Semiconductors, 52, 1791 (2018).

[7] P.A. Belov, E.S. Khramtsov, J. Phys.: Conf. Ser., 816, 012018 (2017).

[8] P.S. Grigoryev, A.S. Kurdyubov, M.S. Kuznetsova et al., Superlatt. Microstr., 97, 452 (2016). 